\title{
Evaluation and Comparison of the Maxillary Palatal Volume of the Unilateral Cleft Lip and Palate Cases Treated with and without Presurgical Naso-Alveolar Molding as Compared to the Non-Cleft Children - A 3D DVT Study
}

\author{
Pallavi Sachin Daigavane (Thombare) ${ }^{1}$, Sunita Shrivastav², Priyanka Niranjane ${ }^{3}$, \\ Rizwan Gilani' ${ }^{4}$, Ranjit Kamble ${ }^{5}$, Bhagyashri Chimote ${ }^{6}$ \\ 1,2,3,4,5 Department of Orthodontics and Dentofacial Orthopaedics, Sharad Pawar Dental \\ College, Sawangi, Wardha, Maharashtra, India, ${ }^{6}$ Department of Prosthodontics, Vidarbha, \\ Youth Welfare Society's Dental College and Hospital, Amravati, Maharashtra, India.
}

\section{ABSTRACT}

\section{BACKGROUND}

Cleft individuals have a significant altered growth of the craniofacial structures since birth. The treatments available for infants are mostly associated with controversies regarding timing of initiation, types \& timing in surgery, grafting etc. Maxilla is affected in all three dimensions; due to surgery alone or in conjugation with the infant orthopaedics, intrinsic growth retardation or combination of all. Considering the current conflict over the use of pre-surgical nasoalveolar molding (PNAM) and to evaluate the efficacy of PNAM technique, this postdoctoral research was done to compare the maxillary palatal volume in unilateral cleft lip and palate (UCLP) cases treated with and without PNAM as compared to non-cleft individuals.

\section{METHODS}

This is an observational cross-sectional study. The palate alveolar volume was assessed and analysed using 3D-DVT angiography machine.

\section{RESULTS}

There was significant difference between cleft group and non-cleft individuals. The cases treated with PNAM had lesser palatal volume.

\section{CONCLUSIONS}

Restrictive effect on maxilla was evident in PNAM cases, but this altered growth could also be an amalgamation of numerous factors like surgery, infant orthopaedics, and intrinsic growth. Therefore, judicious use, current concepts, and biomechanics of PNAM is the need of an hour.

\section{KEY WORDS}

Maxillary Palatal Volume, Unilateral Cleft Cases, Presurgical Naso-Alveolar Molding, 3D-DVT
Corresponding Author: Dr. Pallavi Sachin Daigavane(Thombare), 102, Department of Orthodontics and Dentofacial Orthopaedics, Sharad Pawar Dental College, Sawangi, Wardha - 442001, Maharashtra, India.

E-mail: pallavi.daigavane15@gmail.com

DOI: $10.14260 /$ jemds/2021/542

How to Cite This Article:

Daigavane (Thombare) PS, Shrivastav $S$, Niranjane $P$, et al. Evaluation and comparison of the maxillary palatal volume of the unilateral cleft lip and palate cases treated with and without presurgical nasoalveolar molding as compared to the noncleft children - a 3d DVT study. J Evolution Med Dent Sci 2021;10(32):2652-2657, DOI: $10.14260 /$ jemds $/ 2021 / 542$

Submission 19-11-2020,

Peer Review 01-06-2021,

Acceptance 09-06-2021,

Published 09-08-2021.

Copyright (C) 2021 Pallavi Sachin Daigavane (Thombare) et al. This is an open access article distributed under Creative Commons Attribution License [Attribution 4.0 International (CC BY 4.0)] 


\section{BACKGROUND}

Cleft of the lip, alveolus and palate are the most common congenital malformations trailing clubfoot ${ }^{1}$ in incidence that presents a significant health problem with a greater challenge to healthcare professionals. It is important to understand the aetiology and growth in cleft before planning any treatment strategy. Major concern amongst the unfavourable sequel of the cleft is related to the growth and development of the craniofacial structure. Most commonly affected skeletal tissue of the head, neck and face region is the maxilla with its counter effects on the cranial base and the mandible. ${ }^{2}$

Cleft requires a long term management from birth to puberty for which there are various protocols advised and followed by many centres across the globe. Amongst all the widely accepted protocols are the European cleft and American Cleft. With modification in the basic protocols, various centres treat their cases, but none of the protocols till date has proved itself to be beneficial over other. Shaw $\mathrm{W}^{3}$ in 1992 compared protocols followed in 6 centres and evaluated their benefits at the end of the treatment.

Over the years, numerous treatment modalities have been attempted so as to achieve satisfactory outcome which includes presurgical orthopaedics, lip and palate repair techniques, mid facial growth deficiency corrections, fistula closures with bone grafting, surgical revisions and orthognathic surgeries with severe skeletal discrepancies. In spite of the research, time and effort invested, achieving optimal aesthetics and restoring ideal functions continue to pose a challenge to the concerned professionals. Initially surgical repair was the only choice. To further improve the aesthetics and functions the concept of presurgical infant orthopaedics was developed for the cleft neonates.

$\mathrm{McNeil}^{4}$ in 1959 described the modern concept of presurgical maxillary orthopaedics. From McNeil's concept of alveolar molding to nasoalveolar molding numerous changes in appliance design and concepts have arrived, amongst which Presurgical Nasoalveolar Molding (PNAM) gained fame worldwide. PNAM (a passive appliance) was introduced by Grayson $^{5}$ after Matsuo ${ }^{6}$ stated the importance of molding of the nasal cartilage. PNAM was basically introduced to reshape the alveolar and nasal segments prior to surgical repair. Every technique is associated with its pros and cons so does the PNAM technique. Literature has varied data on long term effects of the PNAM technique with positive and negative effects ${ }^{7}$ with an unsolved controversy still existing.

The proponents for the PNAM technique claim that, this non-surgical treatment molds the alveolar segments into better arch form, prevents collapse, improves dento-maxillary development and maxillomandibular relationship, and primary surgical repair of nose. Lip seals under minimum tension, reduces the scar formation ${ }^{8}$ which reduces the severity of the defect, improves nasal symmetry, nasolabial aesthetics without detrimental effects on mid facial growth ${ }^{9,10}$ and helps in psychological wellbeing of parents. The appliance guides the tongue for normal function thus promoting a guided growth. According to the opponents, surgery alone can suffice the requirement for facial symmetry while PNAM has constrictive effects on the arch in transverse and sagittal dimensions. It retards the growth of the palatal tissue, and has no long term effect on facial appearance. ${ }^{11}$

The controversy deals around three dimensional alterations in growth of maxilla. The degree of collapse is influenced by genetic, environmental as well as iatrogenic effects of surgical interventions, with an additive effect of PNAM.7 There are studies which state that; severity of cleft affects the maxillary growth while the initial cleft size has no role to correlate with the treatment outcome.12 Therefore, WHO has given specific guidelines to treat the PNAM cases. ${ }^{13}$ Considering the current conflict, an observational crosssectional post-doctoral research study was planned to evaluate and compare the maxillary palatal volume of the unilateral cleft cases treated with and without PNAM as compared to the non-cleft children.

\section{METHODS}

After the institutional ethical committee approval, the observational, cross-sectional study was conducted in the Department of Orthodontics, SPDC and Department of Radiology, AVBRH from 2011 to 2015 for a period of 4 years. The need of the study and design was explained to the parents and written consent was obtained. For the sample size estimation, the method of sampling used was LaMorte Power calculation. ${ }^{14}$ The study sample consisted of 48 subjects which were divided into 3 groups:

Group I:(N =16) 7 - 9 years aged non syndromic UCLP cases, were treated with Grayson's PNAM technique from 2006 to 2009, where the treatment was initiated around 7 to 60 days. The lip was operated at $6-7$ months by Millard's technique except in one case which was operated by Z-plasty. Palate was operated at $14-17$ months with Veau Wardill Kilner Push Back technique by same surgical team. These cases had never undergone primary rhinoplasty, primary alveolar bone grafting or gingivo perioplasty.

During these 7 years of follow up they were simultaneously evaluated for the maxillary arch form, 8 stability of nasal and facial symmetry correction, speech ${ }^{15}$ and quantitative evaluation of maxillary hypoplasia in the form of postgraduate thesis.

Group II:(N =16) 7 - 9 years aged non syndromic UCLP cases who were never treated with PNAM, but the lip was operated at around $6-7$ months and palate around $14-17$ months.

Group III: $(\mathrm{N}=16) 7-9$ years aged normal non cleft children.

To assess the palatal volume, the study sample were subjected for 3D-DVT imaging in the TIFAC Cath lab using Phillips Allura X per FD20 3DRA, Digital subtraction Angiography unit (Netherland) with exposure parameters of $80 \mathrm{kvp}, 10 \mathrm{MA}$ and $4-5 \mathrm{sec}$ exposure with the field view 12" 270 degree rotations (Fig 1). Three dimensional virtual images of the skull were reconstructed on a computer with Inturis Suite R2 software. The skull was set up as the specification of the software. These virtual models were first positioned in a standardized way from the lateral and frontal directions. Landmarks were chosen to characterize and measure the spatial positioning of maxilla, which was then separated from 
the other skeletal apparatus with the help of image modifier. Reference point was taken as suggested by Grummon in his analysis. On frontal view a horizontal line was drawn from point J to J (deepest point on the maxillary buttress) on the right and left side passing through or just above the nasal floor and the skull was separated from the maxilla On the palatal view a horizontal line was drawn from the most posteriorly erupted teeth near the tuberosity passing through the PNS projection and thus the posterior or basal skull was separated.

The volume assessment tool was used to fit into the maxilla, wherein the volumetric sphere in the palatal vault was adjusted in frontal posterior, anteroposterior (Fig. 1), occlusal (Fig. 2) and transverse direction. The images were stored as JPEG after a snap shot. The measurements were repeated by observer 2 to reduce the bias and the values obtained were subjected to the reliability analysis (Table 1 ).

\section{Statistical Analysis}

Data was analysed using SPSS 17.0 version and $P<0.05$ was considered as level of significance.

\section{RESULTS}

The agreement between the two observers was found significant i.e., $95 \%$ in the cases treated with PNAM, $98 \%$ in the cases treated without PNAM and $98 \%$ in the non-cleft group (Table 1). Thus the measurement error was found minimum. The mean palate alveolar volume in cases treated with PNAM was $2.29 \pm 0.91$, in cases treated without PNAM was $3.44 \pm 0.85$ and in group non-cleft children it was $3.68 \pm$ 0.94 .

\begin{tabular}{|ccc|}
\hline Group & Reliability & P - Value \\
Cases treated with PNAM & 0.954 & $0.000, \mathrm{~S}, \mathrm{P}<0.05$ \\
Cases treated without PNAM & 0.982 & $0.000, \mathrm{~S}, \mathrm{P}<0.05$ \\
Non-cleft children & 0.980 & $0.000, \mathrm{~S}, \mathrm{P}<0.05$ \\
\hline Table 1. Reliability Analysis between Two Observers \\
\multicolumn{3}{|c}{ - Crohn Bach Alpha test } \\
\hline
\end{tabular}

In the cases treated with PNAM the minimum volume was found to be $1.18 \mathrm{ml}$ while maximum was $3.98 \mathrm{ml}$, in cases treated without PNAM the minimum volume was $1.23 \mathrm{ml}$ while the maximum was $4.81 \mathrm{ml}$, while in the non-cleft children the minimum volume was $2.06 \mathrm{ml}$ while the maximum was $4.80 \mathrm{ml}$ (Table 2).

\begin{tabular}{|c|c|c|c|c|c|c|c|}
\hline Group & $\mathbf{N}$ & Mean & S.D. & $\begin{array}{c}\text { Std. } \\
\text { Error }\end{array}$ & $\begin{array}{c}95 \% \text { Con } \\
\text { Interval fo } \\
\text { Lower } \\
\text { Bound }\end{array}$ & $\begin{array}{l}\text { fidence } \\
\text { or Mean } \\
\text { Upper } \\
\text { Bound }\end{array}$ & P - Value \\
\hline $\begin{array}{l}\text { Cases treated } \\
\text { with PNAM }\end{array}$ & 16 & 2.26 & 0.91 & 0.19 & 1.80 & 2.33 & $0.0012 \mathrm{P}<0.05$ \\
\hline $\begin{array}{c}\text { Cases treated } \\
\text { without PNAM }\end{array}$ & 16 & 3.14 & 0.79 & 0.16 & 2.17 & 4.80 & $0.0019 \mathrm{P}<0.05$ \\
\hline $\begin{array}{l}\text { Non-cleft } \\
\text { children }\end{array}$ & 16 & 3.49 & 0.83 & 0.23 & 2.33 & 4.68 & $0.0421 \mathrm{NS}, \mathrm{P}>0.05$ \\
\hline
\end{tabular}

Comparison of the palato-alveolar volume in three groups was done by the multiple comparative Tukey test which showed statistically significant variation in the three groups. Statistically significant difference was found when the cases treated with PNAM were compared to cases treated without PNAM and non-cleft children. While statistically nonsignificant difference was found when the cases treated without PNAM were compared with the non-cleft children. The palate alveolar volume of the cases treated with PNAM (group I) was lesser than the cases not treated with PNAM (group II) and non - cleft children (group III) (Graph).

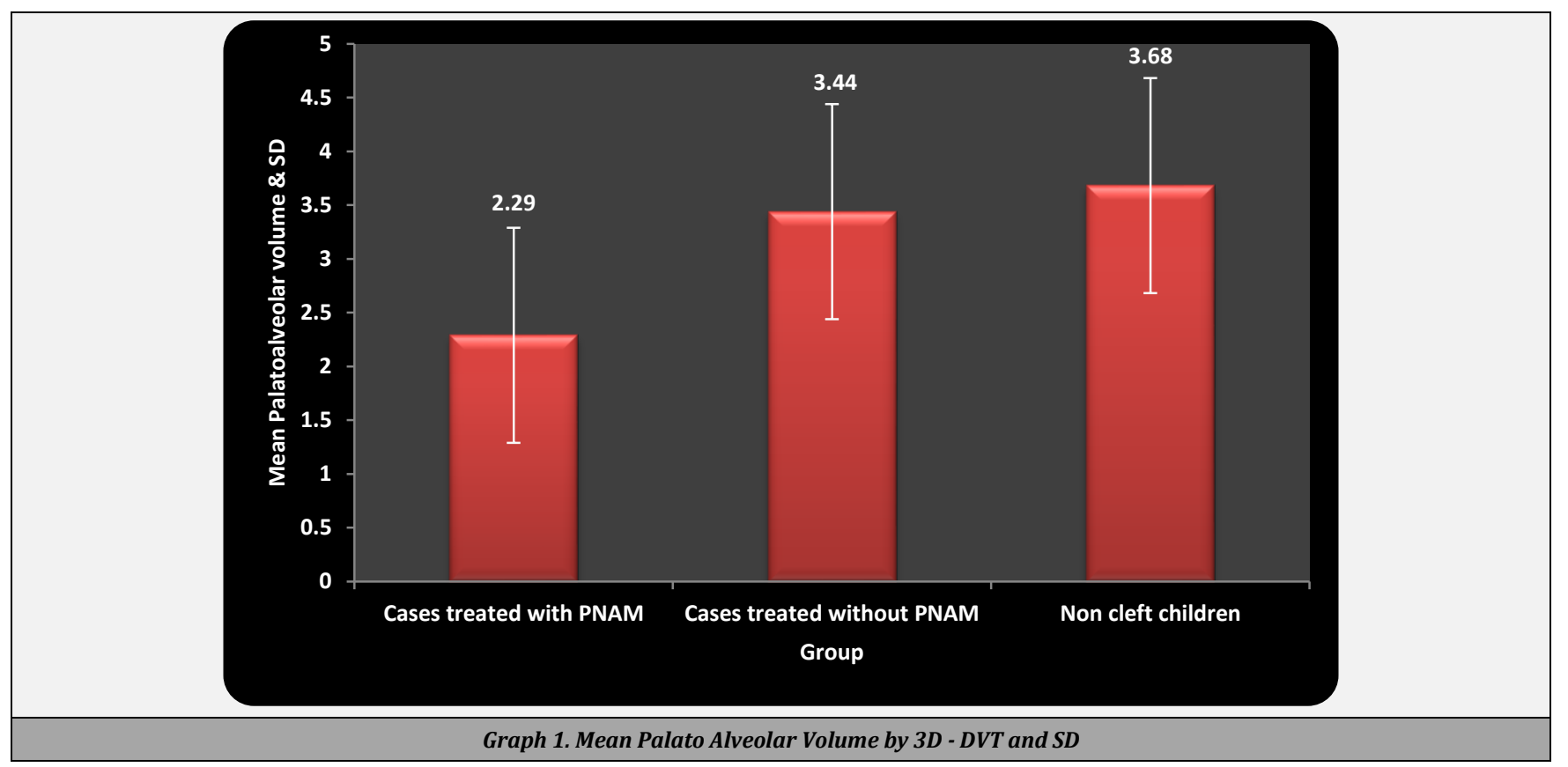



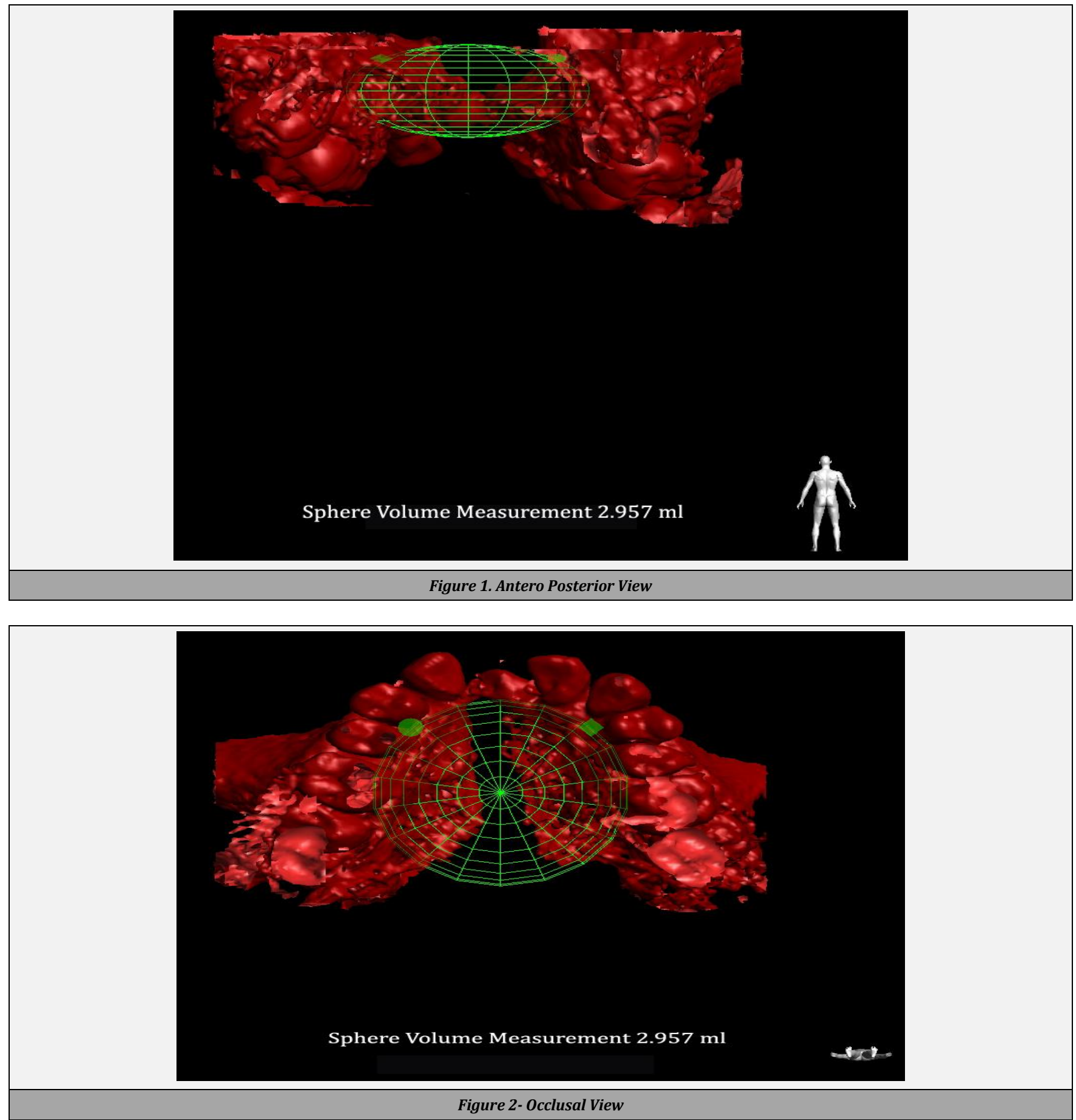

\section{DISCUSSION}

The commonly affected skeletal tissue in the cleft patients is supposed to be "the maxilla". More often, the effects of treatment, particularly surgical technique, timing, and the expertise of the surgeon, have been studied and considered to have a great impact on the altered growth whereas pre surgical orthopaedics and orthodontic treatment also influence the final growth outcome. ${ }^{3}$ Many authors have stated fibrous scar tissue as a major contributing factor for the maxillary hypoplasia. But, the overall growth retardation can be an amalgamation of ${ }^{4}$ surgeries, infant orthopaedics, genetic endowment and the inherent / intrinsic growth of the craniofacial structure. Because of this inconclusive data, cleft is mostly associated with multiple controversies since years.
With respect to the altered maxillary growth, two competing treatment philosophies have raised interest in researchers' worldwide i.e. surgical correction alone versus surgery in conjunction with presurgical orthopaedics.

Out of this quest, to better understand the growth and related alterations in cleft many researchers have evaluated maxilla and craniofacial morphology using innovative methods and various parameters. These evaluations are mostly centred towards dimensional changes and stability of the maxilla pre and post treatment that includes linear and angular measurements. Amongst the literature search, few studies have attempted to evaluate surface area of maxilla; also, there are very limited studies where the palatal volume of an irregular object like maxilla has been evaluated. Thereby with an attempt to evaluate the maxillary palatal volume a newer methodology has been used which is thought to be one 
of its kind. Because of the limited literature available, to evaluate the efficacy of this ideology and methodology, palatal volume evaluation for the same sample was done as per the Archimedes' principle of sand displacement test ${ }^{16}$ wherein both the methods of evaluation of the maxillary volume in the cases treated with PNAM were found to be lesser than the cases not treated with PNAM as compared to non-cleft cases with a significant difference.

The reason associated with the lesser volume in group I was not evaluated in the present study except that they were treated with PNAM. The reason behind this finding could be the associated drawback with PNAM technique which is the over closure of the cleft defect for surgical benefits like nasal floor construction and minimum tension during lip and palatal closure. Over enthusiastic use of molding technique to close the cleft defect, sometimes molds the larger segment palatally rather than molding the lesser segment buccally and labially which can further lead to arch constriction. The previous assessments on the PNAM treated cases in the department gave more over positive results with respect to the evaluation of maxillary arch dimension, nasal and facial symmetry and speech. The results obtained from this study motivated us to critically analyse where, what went wrong with the technique. From the retrospective data, pre-treatment casts were assessed for the defect size and severity, length, size, position and correlation of the alveolar segments, etc. It was noticed that there were some limitations associated while treating the severe cleft defects with PNAM, therefore proper case selection, age of initiation, assessment of defect severity,17,18 knowledge regarding presurgical orthopaedic biomechanics ${ }^{18}$ and parent cooperation should be considered.

There are numerous literature 7,19 which includes clinical trials, review, and meta-analysis where the benefits and efficacy of the PNAM technique have been evaluated. Few have stated the disadvantages of PNAM on the maxillary growth retardation, but the associated advantages include nasal and facial symmetry with long term retention, an alternative aid for suckling and swallowing during feeding, improvement in speech, understanding ability, reduction in hypernasality and nasal air emission, ${ }^{15}$ and above all a psychological wellbeing for parents and patients which should not be overlooked.

\section{CONCLUSIONS}

With a lesser palate alveolar volume in cases treated with PNAM than those which were not treated with PNAM, it is suggested that a judicial and judgmental use of PNAM is necessary for selecting cleft cases for PNAM therapy.

Data sharing statement provided by the authors is available with the full text of this article at jemds.com.

Financial or other competing interests: None.

Disclosure forms provided by the authors are available with the full text of this article at jemds.com.

\section{REFERENCES}

[1] Bailey BJ, Johnson JT, Newlands SD. Head and neck surgery otolaryngology. Philadelphia: Lippincott-Raven 1998.
[2] Ross RB. The clinical implications of facial growth in cleft lip and palate. Cleft Palate J 1970;7:37-47.

[3] Shaw WC, Asher-Mcdade C, Brattstrom V, et al. A six centre study of treatment outcome in patients with cleft of the lip and palate: part 1. Principles and study design. Cleft Palate Craniofac J 1992;29(5):393-7.

[4] McNeil CK. Orthodontic procedures in the treatment of congenital cleft palate. Dent Rec 1950;70(5):126-32.

[5] Grayson BH, Shetye PR. Presurgical naso-alveolar molding treatment in cleft lip and palate patients. Indian J Plast Supplement 2009;42(Suppl):S56-61.

[6] Matsuo K, Hirose T, Tonomo T. Nonsurgical correction of congenital auricular deformities in the early neonate: a preliminary report. Plast Reconstr Surg 1984;73(1):3850.

[7] Niranjane P, Kamble R, Diagavane P, et al. Current status of presurgical infant orthopaedic treatment for cleft lip and palate patients: a critical review. Indian J Plast Surg 2014;47(3):293-302.

[8] Sabarinath P, Daigavanae P, Hazarey P, et al. Changes in maxillary alveolar morphology with naso-alveolar molding. J Clinical Pead Dent 2010;35(2):207-12.

[9] Barillas I, Dec W, Warren S, et al. Naso alveolar molding improves long term nasal symmetry in complete unilateral cleft lip-cleft palate patients. Plast Reconstr Surg 2009;123(3):1002-6.

[10] Pai BCJ, Ko EWC, Huang CS, et al. Symmetry of the nose after presurgical naso-alveolar molding in infants with unilateral cleft lip and palate: a preliminary study. Cleft Palate Craniofac J 2005;42(6):658-63.

[11] Prahl C, Prahl-Anderson B, A Van't Hof M, et al. Infant orthopedics and facial appearance: a randomized clinical trial (Dutchcleft). Cleft Palate Cranoiofac J 2006;43(6):659-64.

[12] Peltomaki T, Vendittelli D, Grayson B, et al. Association between severity of cleft and maxillary growth in patients with unilateral cleft lip and palate treated with infant orthopedics. Cleft Palate Craniofac J 2001;38(6):582-90.

[13] Guidelines for the presurgical naso alveolar molding in complete cleft lip and palate cases. WHO Publications 2001.

[14] Lwanga SK, Lemeshow S. Sample size determination in health studies: a practical manual. Geneva: WHO Publication 1991.

[15] Thomas R, Daigavane P, Shrivastav S. Assessment of role of palatal dimensions on speech in cleft lip and palate patients treated with and without presurgical orthopedics. IOSR-Journal of Dental and Medical Sciences 2016;15(8):96-104.

[16] Daigavane PS, Shrivastava S, Kamblerh, et al. Sand displacement test-a newer strategy to evaluate maxillary palaal volume of the unilateral cleft cases treated with and without presurgical naso alveolar molding as compared to normal children. IJDMHS 2016;3:17-27.

[17] Al-Gunaid T, Asahito T, Yamaki M, et al. Relapse tendency in the maxillary arch width in unilateral cleft lip and palate patients with different maxillary arch forms. Cleft Palate Craniofac J 2008;45(3):278-83.

[18] Daigavane PS, Hazarey PV, Niranane P, et al. A new classification system for unilateral cleft lip and palate infants to assist presurgical infant orthopedics. J Clin Pediatr Dent 2015;39(3):230-33. 


\section{Jemds.com}

[19] Murthy PS, Deshmukh S, Bhagyalakshmi A, et al. Pre surgical naso alveolar molding: changing paradigms in
Original Research Article

early cleft lip and palate rehabilitation. J Int Oral Health 2013;5(2):70-80. 